\title{
No Association Between Screening for Hepatocellular Carcinoma and Reduced Cancer-Related Mortality in Patients With Cirrhosis
}

\author{
Andrew M. Moon, ${ }^{1}$ Noel S. Weiss, ${ }^{5}$ Lauren A. Beste, ${ }^{3}$ Feng Su, ${ }^{2}$ Samuel B. Ho, ${ }^{6}$ \\ Ga-Young Jin, ${ }^{4}$ Elliott Lowy, ${ }^{4}$ Kristin Berry, ${ }^{4}$ and George N. Ioannou ${ }^{2,4}$ \\ ${ }^{1}$ Division of Gastroenterology and Hepatology, University of North Carolina School of Medicine, Chapel Hill, North Carolina; \\ Divisions of ${ }^{2}$ Gastroenterology and ${ }^{3}$ General Internal Medicine, Veterans Affairs Puget Sound Healthcare System and University \\ of Washington, Seattle, Washington; ${ }^{4}$ Research and Development, Veterans Affairs Puget Sound Healthcare System, Seattle, \\ Washington; ${ }^{5}$ Department of Epidemiology, University of Washington, Seattle, and Fred Hutchinson Cancer Research Center, \\ Seattle, Washington; and ${ }^{6}$ Division of Gastroenterology, Veterans Affairs San Diego Healthcare System and University of \\ California, San Diego, California
}

This article has an accompanying continuing medical education activity, also eligible for MOC credit, on page e17. Learning Objective: Upon completion of this CME activity, successful learners will be able to recognize current screening and treatment guidelines for hepatocellular carcinoma (HCC), describe gaps in existing evidence supporting HCC screening, and explain how unique research methodologies, including the case-control study design, addresses these voids.

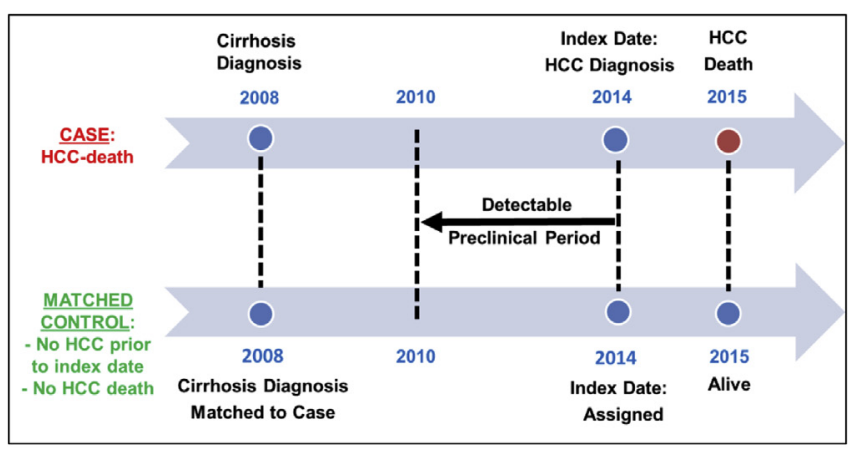

BACKGROUND \& AIMS: Screening patients with cirrhosis for hepatocellular carcinoma (HCC) has been recommended. We conducted a matched case-control study within the US Veterans Affairs (VA) health care system to determine whether screening by abdominal ultrasonography (USS) and/or by measuring serum level of $\alpha$-fetoprotein (AFP) was associated with decreased cancer-related mortality in patients with cirrhosis. METHODS: We defined cases $(n=238)$ as patients with cirrhosis who died of HCC from January 1, 2013 through August 31, 2015 and had been in VA care with a diagnosis of cirrhosis for at least 4 years before the diagnosis of HCC. We matched each case to 1 control $(n=238)$, defined as a patient with cirrhosis who did not die of HCC and had been in VA care for at least 4 years before the date of the matched case's HCC diagnosis. Controls were matched to cases by year of cirrhosis diagnosis, race and ethnicity, age, sex, etiology of cirrhosis, Model for End-Stage Liver Disease score, and VA medical

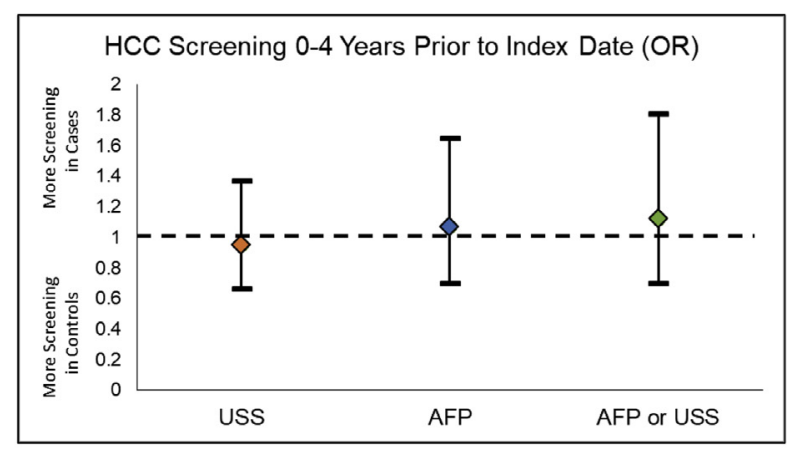

\section{Gastroenterology}

center. We identified all USS and serum AFP tests performed within 4 years before the date of HCC diagnosis in cases or the equivalent index date in controls and determined by chart extraction (blinded to case or control status) whether these tests were performed for screening. RESULTS: There were no significant differences between cases and controls in the proportions of patients who underwent screening USS $(52.9 \%$ vs $54.2 \%)$, screening measurement of serum AFP $(74.8 \%$ vs $73.5 \%)$, screening USS or measurement of serum AFP $(81.1 \%$ vs $79.4 \%$ ), or screening USS and measurement of serum AFP ( $46.6 \%$ vs $48.3 \%$ ) within 4 years before the index date, with or without adjusting for potential confounders. There also was no difference in receipt of these screening tests within 1,2 , or 3 years before the index date. CONCLUSIONS: In a matched casecontrol study of the VA health care system, we found that screening patients with cirrhosis for HCC by USS, measurement of serum AFP, either test, or both tests was not associated with decreased HCC-related mortality. We encourage additional case-control studies to evaluate the efficacy of screening for HCC in other health care systems, in which available records 
are sufficiently detailed to enable identification of the indication for USS and AFP tests.

Keywords: Surveillance; Survival; Liver Cancer; Liver Transplantation.

D atients with cirrhosis have a high risk of hepatocellular carcinoma (HCC), ranging from $1 \%$ to $8 \%$ per year. ${ }^{1}$ Most professional liver societies recommend screening patients with cirrhosis with abdominal ultrasonography (USS) with or without concomitant serum $\alpha$-fetoprotein (AFP) testing every 6 months, ${ }^{2-4}$ but many non-liver societies do not endorse HCC screening. ${ }^{5,6}$ The rationale for HCC screening in patients with cirrhosis is that screening tests such as USS or serum AFP could identify patients with HCC at an early stage when they have potentially curative or life-prolonging treatment options, including liver transplantation, radiofrequency ablation, or surgical resection. However, it remains unclear whether HCC screening decreases cancer-related mortality in patients with cirrhosis, which should be the primary end point of HCC screening, rather than early-stage migration or increased frequency of receipt of potentially curative treatments.

Two randomized controlled trials (RCTs) of HCC screening have been performed. ${ }^{7,8}$ However, these trials reached conflicting conclusions about screening effectiveness, and their methodology has been criticized. ${ }^{9}$ Also, their results do not necessarily apply to North American and European patients with cirrhosis in the current era, because the trials were conducted in China from 1989 to 1997 in patients with chronic hepatitis B virus infection. HCC related to hepatitis B virus can occur in the absence of cirrhosis and important advances in the treatment of HCC have occurred since these studies were conducted.

Many observational studies have compared survival in patients diagnosed with HCC by screening with those who presented with symptomatic HCC. These studies were summarized in 2 systematic reviews, ${ }^{9,10}$ which concluded that the interpretation of these observational studies was limited because of selection, verification, and lead-time and length-time biases.

Ideally, the effectiveness of HCC screening would be evaluated by a study that randomizes patients with cirrhosis to screening vs no screening. However, as concluded by the authors of the American Association for the Study of Liver Diseases (AASLD) HCC guidelines ${ }^{11}$ and demonstrated by problems in patient recruitment encountered in a pilot study, ${ }^{12}$ it is unlikely that such randomized trials of HCC screening will be feasible in the United States, where HCC screening has become the de facto standard of care. Nonetheless, concerns have been raised that HCC surveillance has been adopted in the United States without sufficient data to demonstrate its efficacy. ${ }^{13,14}$

As an alternative to RCTs, case-control studies have the potential to evaluate the effectiveness of cancer screening in an efficient manner. ${ }^{15-17}$ To test for an effect of screening on cancer-related mortality, previous receipt of the screening

\section{WHAT YOU NEED TO KNOW}

\section{BACKGROUND AND CONTEXT}

The matched case-control study design is the best observational study design for determining whether screening reduces cancer-related mortality. A casecontrol study of HCC screening in patients with cirrhosis has not yet been performed.

\section{NEW FINDINGS}

HCC screening with ultrasonography, AFP, or both was not associated with decreased HCC-related mortality. This contrasts with many "cohort studies" of HCC screening, which are susceptible to lead-time and length-time bias.

\section{LIMITATIONS}

The main limitation of case-control studies of screening effectiveness is misclassification of tests performed among cases for suspected cancer as screening tests.

\section{IMPACT}

Current strategies for HCC screening have been based on ultrasonography \pm AFP for more than 25 years. The authors hope that this study will lead to renewed efforts to develop and validate better screening tests.

test (eg, abdominal USS or serum AFP testing) was compared in patients with cirrhosis who died of HCC (cases) and in a matched sample of patients with cirrhosis who did not die of HCC (controls). A lower likelihood of screening before diagnosis during the time when the malignancy is occult but potentially detectable by the screening modality in those who die of cancer would provide evidence in support of a protective effect of screening on mortality. Thus, if HCC screening were effective, then we would expect patients who died of HCC to be less likely to have been screened than patients with cirrhosis who did not die of HCC. By selecting patients with fatal, rather than incident, cancers as case subjects, this case-control paradigm addresses the impact of screening on cancer-related mortality and is not susceptible to length-time or lead-time bias. The odds ratio (OR) in a bias-free case-control study of screening would be a valid estimate of the risk ratio that might be obtained from an RCT. ${ }^{15}$

The case-control study design has been used previously to evaluate screening effectiveness for malignancies other than HCC, such as colorectal cancer, ${ }^{18,19}$ breast cancer, ${ }^{20}$ esophageal cancer, ${ }^{21}$ cervical cancer, $^{22}$ prostate cancer, ${ }^{23}$

Abbreviations used in this paper: AASLD, American Association for the Study of Liver Diseases; AFP, $\alpha$-fetoprotein; CAPRI, Compensation and Pension Record Interchange; CI, confidence interval; CDW, Corporate Data Warehouse (Veterans Affairs); CT, computed tomography; DPP, detectable preclinical phase; HCC, hepatocellular carcinoma; HCV, hepatitis C virus; ICD-9, International Statistical Classification of Diseases, Injuries and Causes of Death, Ninth Edition; MELD, Model for End-Stage Liver Disease; MRI, magnetic resonance imaging; OR, odds ratio; RCT, randomized controlled trial; USS, ultrasound scan; VA, Veterans Affairs. 


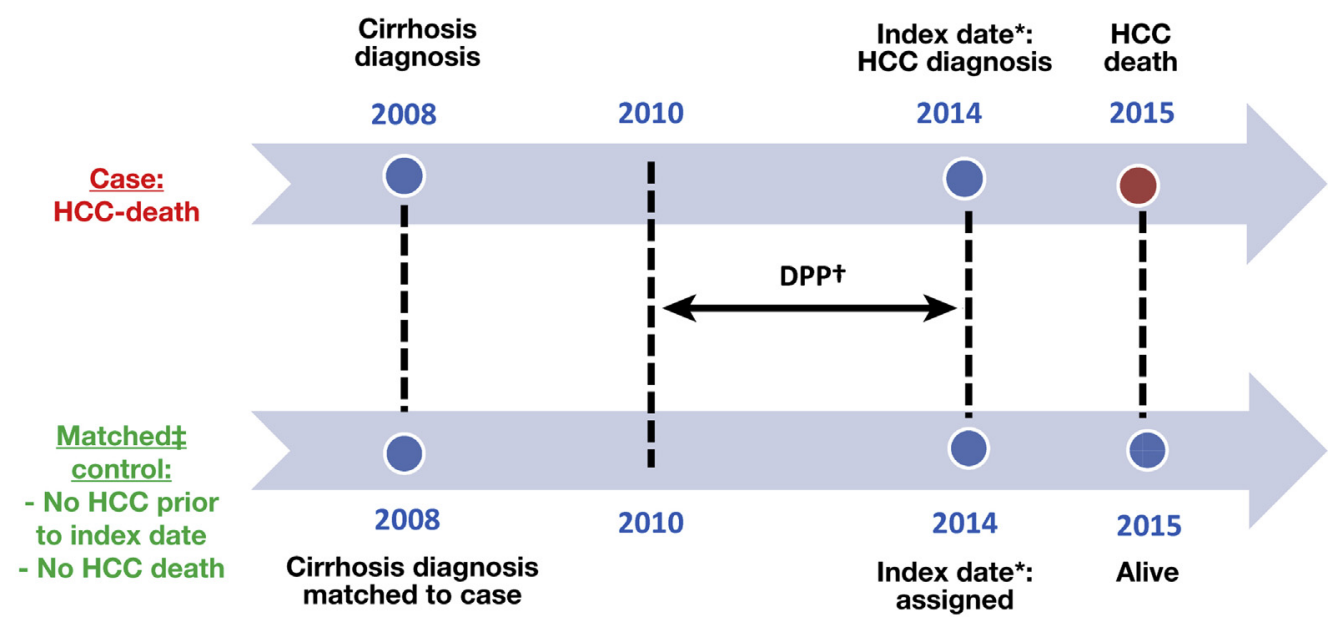

Figure 1. Schematic representation of the case-control study design, illustrating the criteria used to match cases (fatal $\mathrm{HCC}$ ) to controls, the index date, and the DPP. The DPP consisted of an identical period of calendar years for the case and control within each matched pair (eg, 2010-2014 in the example), during which the case and control were in VA care at the same VA facility. *Index date is the date of HCC diagnosis or the earliest date that patients showed symptoms, laboratory abnormalities, or imaging findings suspicious for HCC. ${ }^{\dagger}$ The DPP is the period before the index date during which we documented the occurrence of screening USS or serum AFP. ${ }^{\ddagger}$ Cases and controls were matched by age, sex, race, etiology of cirrhosis, MELD score at the time of cirrhosis diagnosis, date of cirrhosis diagnosis, and VA facility.

and melanoma. ${ }^{24}$ We performed a matched case-control study to evaluate the extent to which screening for HCC with USS or serum AFP would be associated with decreased HCC-related mortality in patients with cirrhosis in the US Veterans Affairs (VA) health care system, the largest integrated health care system in the United States.

\section{Methods}

\section{Overall Study Design: Matched Case-Control Study of Screening Effectiveness}

We defined cases as VA patients with cirrhosis who died of HCC from January 1, 2013 through August 31, 2015 and had at least 4 years of follow-up time enrolled in the VA from the date of cirrhosis diagnosis to the date of HCC diagnosis. We matched each case to 1 control, defined as a VA patient with cirrhosis who did not die of HCC, was not diagnosed with HCC as of the date of the matched case's HCC diagnosis, and was in VA care at least 4 years before the date of the matched case's HCC diagnosis (Figure 1). Cases were compared with controls for abdominal USS or serum AFP tests performed for HCC screening during the 4 years before the diagnosis of HCC in cases or the equivalent index date in matched controls. A smaller proportion of cases than controls receiving HCC surveillance would suggest an association between HCC surveillance and decreased HCC-related mortality.

\section{Data Sources: National VA Corporate Data Warehouse and Medical Chart Extraction}

The VA uses a single, nationwide, comprehensive electronic health care information network. Data from this network reside on the Corporate Data Warehouse (CDW), a national, continually updated data repository developed specifically to facilitate research. ${ }^{25}$ We extracted data on all pharmacy prescriptions, demographics, inpatient and outpatient visits, problem lists, procedures, vital signs, diagnostic tests, and laboratory tests for patients with a diagnosis of cirrhosis who were in VA care during or before 2015. These CDW data were used only to identify potential cases and controls for this study. Once potential cases and controls were identified from the CDW, their electronic medical records were accessed using the Compensation and Pension Record Interchange (CAPRI), an electronic interface providing online access to veterans' medical records at all VA facilities in the country. The CAPRI was used to obtain radiology reports, pathology reports, and inpatient and outpatient progress notes. These detailed records were electronically copied onto a specifically designed REDCap $^{26}$ database. The extracted records were reviewed by a physician-investigator blinded as case-control status.

\section{Identification of Cases: Patients With Fatal HCC}

Identification and confirmation of cases was a 2-step process (Figure 2). First, potential cases were identified electronically from the CDW as patients with a diagnosis of cirrhosis based on appropriate International Classification of Diseases, Ninth Edition (ICD-9) codes (Supplemental Table 1) recorded at least twice ${ }^{27-33}$ who were diagnosed with HCC at least 4 years after the diagnosis of cirrhosis, died from January 1, 2013 through August 31, 2015, and had a Model for EndStage Liver Disease (MELD) score lower than 20 at all times before the HCC diagnosis. We used the presence of $I C D-9$ code 155.0 (primary liver cancer) recorded at least twice for this preliminary identification of HCC, as in previous studies..$^{29,30,33-37}$ A 4-year period was chosen to allow enough time for screening to plausibly have an influence on HCC-related mortality. The interval 2013-2015 was selected because it was the most recent at the time the study was initiated, such that the most "current" treatments would be available to patients diagnosed with HCC. Patients with a MELD score of at least 20 were excluded because screening is not recommended in patients with advanced liver dysfunction (unless they are listed for 


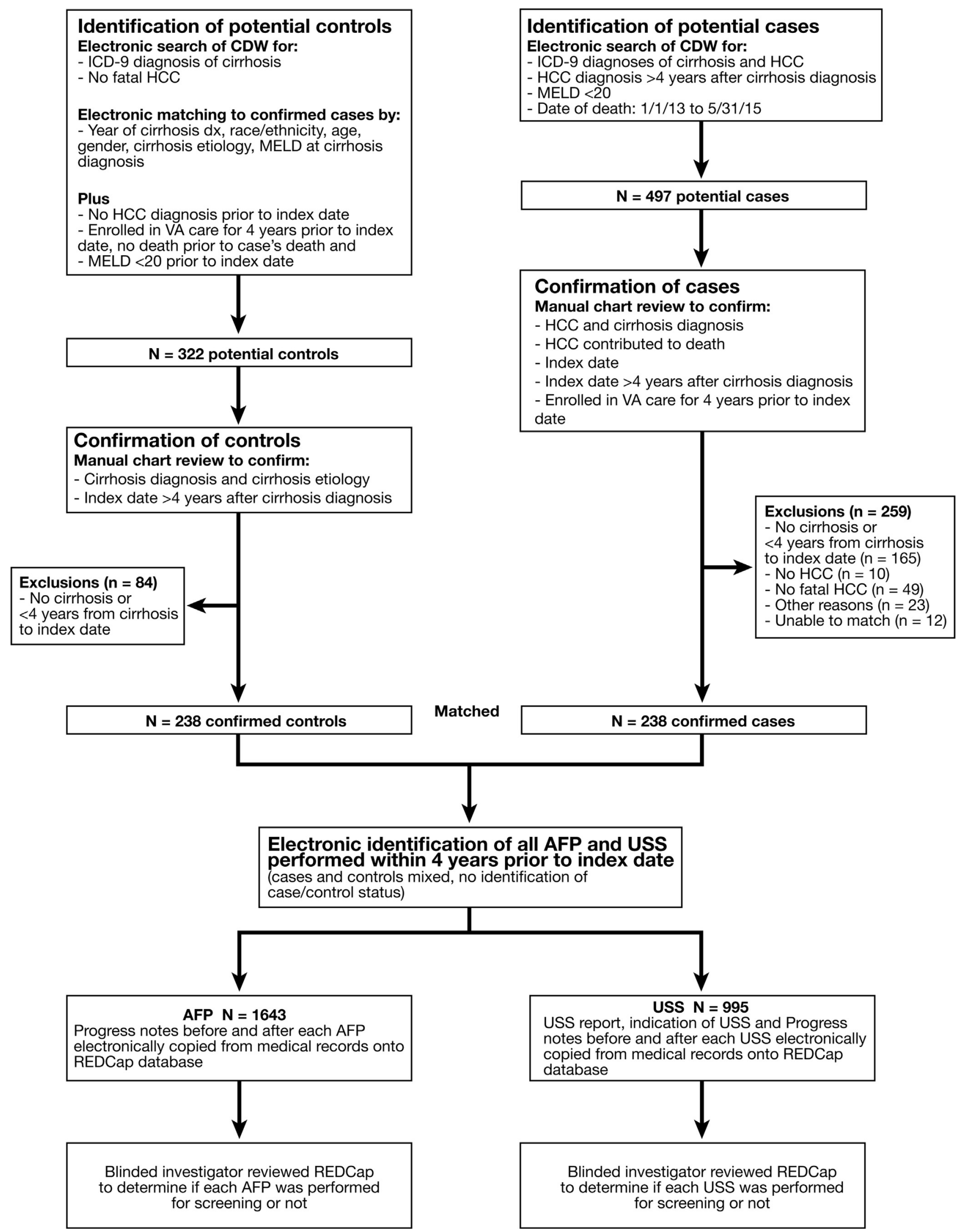

Figure 2. Flowchart demonstrating the identification and confirmation of cases, identification and confirmation of controls and matching to cases, and identification of USS and serum AFP tests performed before the index date. 
liver transplantation). Including such patients might have biased the results in the direction of not finding an association between screening and decreased cancer-related mortality. Second, the medical records of potential cases were accessed at all VA facilities nationally through the CAPRI by a physicianinvestigator blinded to screening status to confirm the diagnosis of cirrhosis and HCC, identify the patients in whom HCC contributed to the patients' death, and determine the index date. The diagnosis of cirrhosis was based on clinical features of portal hypertension from liver disease (ascites, hepatic encephalopathy, varices), characteristic laboratory features (eg, decreased platelets, prolonged prothrombin time, increased serum bilirubin, hypoalbuminemia), imaging characteristics (eg, nodular liver, portosystemic collaterals), liver biopsy results, and/or diagnosis documented by a gastroenterologist or hepatologist. The diagnosis of HCC was defined by the following national AASLD criteria that were in effect at the time our study was conducted ${ }^{11,38}$ : (1) liver nodules at least $10 \mathrm{~mm}$ that were hypervascular in the arterial phase with washout in the portal venous or delayed phase at 4-phase multidetector computerized tomography (CT) or dynamic contrast-enhanced magnetic resonance imaging (MRI);

(2) liver nodules that fulfilled the 5 criteria of the Liver Imaging Reporting and Data System; or (3) liver lesions with histology consistent with HCC at biopsy examination.

For patients confirmed to have HCC, the physicianinvestigator determined whether HCC definitely contributed to the patient's death, which was defined as presence of metastatic HCC, multifocal HCC ( $>3$ lesions), local or vascular invasion by HCC, large-volume HCC $(>6 \mathrm{~cm})$, serum AFP level higher than $1,000 \mathrm{ng} / \mathrm{mL}$, or death from complications from HCC treatment in patients who did not have an obvious alternative cause of mortality. Only patients confirmed to have HCC in whom HCC "definitely" contributed to death were included as cases in the study.

The diagnostic definition of HCC and the criteria used to define that HCC contributed to the patient's death were determined and validated before study initiation by a pilot study of a different set of 50 cases of fatal HCC reviewed independently by 2 of the investigators. There was excellent inter-rater agreement (97.5\% agreement, $\kappa=0.94, P<.001)$ between the 2 investigators in assigning whether HCC definitely contributed to the patient's death using the criteria described earlier.

\section{Identification of Matched Controls}

Identification and confirmation of matched controls was a 2-step process (Figure 2). First, we electronically identified from the CDW all patients with a diagnosis of cirrhosis, ${ }^{27-33}$ defined using the same ICD-9 codes as for cases, who did not die of HCC and were not diagnosed with HCC before their matched case's index date. We matched 1 control to each case by the following characteristics, which are strongly associated with fatal HCC and the likelihood of screening: (1) year of cirrhosis diagnosis; (2) race and ethnicity (categorized as white non-Hispanic, black non-Hispanic, Hispanic, other); (3) age (within 2 years); (4) sex; (5) primary etiology of cirrhosis (hepatitis $\mathrm{C}$ virus [HCV], alcoholic liver disease, nonalcoholic fatty liver disease, or other as previously published ${ }^{39}$; see Supplemental Table 2 for definitions); (6) MELD score at time of cirrhosis diagnosis (within 2 points); and (7) VA facility in which the diagnosis of cirrhosis was made. Controls had to be enrolled in VA care for the 4 years before the index date and alive at the time of their matched case's death. Controls who had a MELD score of at least 20 at any time before the index date of their matched case were excluded (as were cases). Second, the medical records of potential controls were accessed at all VA facilities nationally through the CAPRI by a physicianinvestigator who was blinded to screening status to confirm the diagnosis and etiology of cirrhosis.

\section{Definition of Index Date}

The index date for cases was defined as the date of HCC diagnosis (ie, the earliest date of a multiphasic CT or MRI or tissue biopsy finding diagnostic for HCC) or the earliest date that patients reported symptoms (eg, weight loss, abdominal pain), imaging findings (eg, suspicious liver nodule at screening USS), or laboratory abnormalities suspicious for HCC (eg, increased serum AFP), whichever came first. The index date was determined for each case by review of the medical records by a physician-investigator. By definition, an USS or serum AFP test performed after the index date could not have been a screening test. For example, if a screening USS showed a suspicious liver nodule and a serum AFP was subsequently ordered, the index date was the date of the USS and the serum AFP was not considered a screening test. Each control was assigned the same index date as the matched case. Therefore, for each case-control pair, we evaluated an identical calendar period before the index date for presence of screening USS or AFP (Figure 1). Cases or controls with index dates occurring less than 4 years after the diagnosis of cirrhosis were excluded, because this would not have allowed us to examine screening histories for the full duration of a maximum hypothesized 4-year detectable preclinical phase of HCC.

\section{Determination of Screening USS and Serum AFP in Cases and Controls}

Each abdominal USS test performed within 4 years before the index date was identified electronically by an analyst blinded to case-control status. The USS report (which included the recorded indication for performing the USS) and the ordering provider's progress notes before and after the USS were electronically copied from the medical records onto a REDCap database by a trained research assistant as a separate record for each USS. A physician-investigator blinded to casecontrol status reviewed this information on REDCap and categorized each USS as having been performed "definitely" for screening, "probably" for screening, "probably not" for screening, or "definitely not" for screening. The definitions of these categories are presented in Supplemental Table 3.

This process was performed separately for each serum AFP test obtained within 4 years before the index date. The progress notes of the ordering provider before and after the AFP result were copied from the electronic medical records and a blinded physician-investigator categorized each AFP as having been performed "definitely," "probably," "probably not," or "definitely not" for screening using the criteria listed in Supplemental Table 4.

The principal investigator also reviewed any records that were difficult to categorize by the physician-investigator and a random $10 \%$ sample of all records. 
The criteria for adjudicating the screening status of USS and serum AFPs were determined by an independent chart extraction by 2 investigators of a different set of 50 cases and 50 controls before the study was initiated. There was excellent agreement between the 2 investigators for the criteria used in the study $(94.2 \%$ agreement, $\kappa=0.90, P<.001)$.

The primary analysis considered only USS or serum AFP tests performed "definitely" for screening, but a sensitivity analysis also included those performed "probably" for screening.

\section{Statistical Analysis}

Cases were compared with their matched controls for receipt of abdominal USS or serum AFP performed for screening within $0-1,0-2,0-3$, or $0-4$ years before the index date modeled as binary (yes or no) variables using conditional logistic regression. This period, the detectable preclinical phase (DPP), is the period from the earliest time at which the cancer is potentially detectable using the screening modality under study to the time at which the cancer would present clinically in the absence of screening. Sheu et $\mathrm{al}^{40}$ estimated the DPP for HCC by estimating the time it would take for HCCs to grow from $1 \mathrm{~cm}$ (minimum size potentially detectable by USS) to $10 \mathrm{~cm}$ (a size generally expected to cause symptoms) as 3.2 years for tumors with a median growth rate, which had a doubling time of 117 days. Based on this, we chose 4 years as the upper limit of the DPP, that is, we estimated that a small HCC that could be detectable by USS would take a maximum of 4 years before presenting with clinical symptoms. ${ }^{40}$ The maximal DPP is believed to provide the least biased estimate of any true association between receipt of screening and decreased cancerrelated mortality. ${ }^{41}$ However, it also has been shown that when different periods are analyzed yielding different ORs, the lowest OR (ie, the one that indicates the greatest survival benefit for screening) is likely to be the least biased. ${ }^{41}$ For these reasons, we analyzed screening tests performed within 4 years before the index date (ie, close to the estimated maximal DPP) as our primary analysis, but also analyzed screening tests performed within 1, 2, or 3 years before the index date. We did not analyze tests performed 6 months before the index date, because this short interval would be heavily biased toward showing a higher rate of screening for cases than for controls (ie, erroneously making it appear as if screened patients are more likely to die of HCC).

Cases and controls were not compared for the number of screening tests during the DPP, because even in the absence of effective therapy of screen-detected cancers, the cases would be expected to have been screened fewer times than the controls, assuming that the screening test is sensitive in identifying the tumor, producing a spuriously low OR associated with multiple (or "regular") screening. ${ }^{16,17}$ If a case with occult liver cancer undergoes a screening test, then the cancer might be identified and a second (or third) test will never take place. However, controls (the large majority of whom do not have liver cancer) have the capacity to be screened more than once during the interval under consideration.

Conditional logistic regression models were adjusted for age, etiology of cirrhosis (HCV, nonalcoholic fatty liver disease, alcoholic liver disease, and other), MELD score at cirrhosis diagnosis, race and ethnicity, year of cirrhosis diagnosis, diabetes, alcohol use disorders, body mass index, eradication of HCV by antiviral treatment, and receipt of abdominal CT or MRI during the period of interest. Models that evaluated the effectiveness of screening serum AFP also were adjusted for receipt of screening USS and models that evaluated the effectiveness of screening USS also were adjusted for receipt of screening serum AFP.

We evaluated the following binary screening variables in different conditional logistic regression models:

1. Screening USS vs no screening USS

2. Screening serum AFP vs no screening AFP

3. Screening USS or serum AFP vs no screening with USS or serum AFP

4. Screening with USS and serum AFP vs screening with only USS

5. Screening with USS and serum AFP vs screening with only AFP

6 Screening with USS and serum AFP vs screening with none

\section{Power Calculations}

Extrapolating from a prior VA study, ${ }^{33}$ we estimated that the proportion of controls with a screening serum AFP or a screening USS during a 4-year period in our study would be approximately $70 \%$. Using the method of Dupont ${ }^{42}$ specifically for power calculations in matched case-control studies, we calculated a priori that 238 cases matched to 238 controls would provide more than $90 \%$ power to detect a $14 \%$ difference in screening between cases and controls and more than $80 \%$ power to detect a $12 \%$ difference between cases and controls (eg, 70\% screening for controls and 58\% screening for cases).

\section{Results}

Aiming for a sample size of 238 pairs of cases and matched controls, we initially identified electronically a random sample of 600 potential cases and 1,800 potential matched controls. After reviewing the charts of 497 of these 600 potential cases in random sequence, we excluded 10 patients who did not have HCC, 49 patients in whom HCC did not definitely contribute to patient death, 165 patients who did not have cirrhosis or had an interval shorter than 4 years between the diagnosis of cirrhosis and the index date, 23 patients for other reasons (care elsewhere, $n=4$; insufficient documentation, $\mathrm{n}=8$; unclear cause of death, $\mathrm{n}=8$; unclear HCC diagnosis, $\mathrm{n}=3$ ), and 12 patients who could not be matched to a control who fulfilled all matching criteria, leaving 238 cases in the present analysis (Figure 2). After reviewing the charts of 322 potential controls electronically matched to these cases, we excluded 84 who did not have cirrhosis or had an interval shorter than 4 years between the diagnosis of cirrhosis and the index date, leaving 238 controls in the present analysis, each matched to a single case.

\section{Characteristics of Cases and Controls}

As expected by the matching scheme, cases and controls were the same for age at diagnosis of cirrhosis (54.6 years), age at index date (62.5 years), racial and ethnic distribution, 
Table 1. Characteristics of Cases and Their Matched Controls

\begin{tabular}{|c|c|c|}
\hline & $\begin{array}{l}\text { Controls } \\
(n=238)\end{array}$ & $\begin{array}{c}\text { Cases } \\
(n=238)\end{array}$ \\
\hline Men, \% & 100 & 100 \\
\hline Age at diagnosis of cirrhosis $(y)$, mean & 54.5 & 54.6 \\
\hline Age at index date $(y)$, mean & 61.9 & 62.0 \\
\hline \multicolumn{3}{|l|}{ Year of cirrhosis diagnosis, \% } \\
\hline Before 2003 & 42 & 42 \\
\hline 2003-2005 & 27 & 27 \\
\hline $2006-2008$ & 27 & 27 \\
\hline 2009-2011 & 5 & 5 \\
\hline $\begin{array}{l}\text { Interval from diagnosis of } \\
\quad \text { cirrhosis to index date, } y\end{array}$ & 7.9 & 7.9 \\
\hline \multicolumn{3}{|l|}{ Index date year, \% } \\
\hline$\leq 2012$ & 63 & 63 \\
\hline 2013 & 24 & 24 \\
\hline 2014 & 11 & 11 \\
\hline 2015 & 2.1 & 2.1 \\
\hline \multicolumn{3}{|l|}{ Race, ethnicity, \% } \\
\hline White, non-Hispanic & 74 & 74 \\
\hline Black, non-Hispanic & 15 & 15 \\
\hline Hispanic & 10 & 10 \\
\hline Other & 1.3 & 1.3 \\
\hline \multicolumn{3}{|l|}{ Primary etiology of liver disease, \% } \\
\hline $\mathrm{HCV}$ & 80 & 80 \\
\hline Alcoholic liver disease & 13 & 13 \\
\hline Nonalcoholic fatty liver disease & 2.9 & 2.9 \\
\hline Other & 4.2 & 4.2 \\
\hline $\begin{array}{l}\text { Sustained virologic response } \\
\text { to } \mathrm{HCV} \text { achieved before index } \\
\text { date (in those with } \mathrm{HCV} \text { ), } \%\end{array}$ & 14 & 8.4 \\
\hline $\mathrm{BMI}\left(\mathrm{kg} / \mathrm{m}^{2}\right)$, mean & 29 & 29 \\
\hline MELD score, mean & 9.1 & 9.0 \\
\hline Diabetes, \% & 23 & 23 \\
\hline Alcohol use disorders, \% & 48 & 61 \\
\hline \multicolumn{3}{|l|}{ CT or MRI before index date, \% } \\
\hline $0-2$ y & 44 & 55 \\
\hline $0-3 y$ & 53 & 62 \\
\hline $0-4 y$ & 62 & 71 \\
\hline
\end{tabular}

BMI, body mass index.

year of cirrhosis diagnosis, year of index date, interval from cirrhosis diagnosis to index date, MELD score at time of cirrhosis diagnosis, and primary etiology of cirrhosis (Table 1). All cases and controls were men, reflecting the predominantly male VA population (by chance no women met all the inclusion criteria for cases). In most patients, the primary etiology of cirrhosis was HCV infection $(80 \%)$ or alcoholic liver disease $(13 \%)$. Most patients were white (73.5\%) followed by black (15.1\%) and Hispanic (10.1\%) race and ethnicity. Patients had a mean MELD score of 9 at the time of cirrhosis diagnosis. HCV infection had been cured by antiviral treatment before the index date in $13.7 \%$ of controls and $8.4 \%$ of cases.

\section{Characteristics of HCC in Cases}

In most cases, HCC was diagnosed by appropriate multiphasic CT or MRI (85.6\%), whereas $28.8 \%$ had
Table 2. Characteristics of HCC in Cases (Fatal HCC)

\begin{tabular}{|c|c|}
\hline & Cases, $\mathrm{n}(\%)$ \\
\hline \multicolumn{2}{|l|}{ Method of HCC diagnosis ${ }^{a}$} \\
\hline Imaging (CT or MRI) & $204(86)$ \\
\hline Histology & $69(29)$ \\
\hline \multicolumn{2}{|l|}{ Stage of HCC at diagnosis } \\
\hline $\begin{array}{l}\text { Maximum dimension of largest } \\
\text { tumor }(\mathrm{cm}) \text {, mean (SD) }\end{array}$ & $4.5(3.4)$ \\
\hline Number of tumors, mean (SD) & $2.1(1.6)$ \\
\hline \multicolumn{2}{|l|}{ Number of tumors (\%) } \\
\hline 1 & $125(53)$ \\
\hline $2-3$ & $72(30)$ \\
\hline$\geq 4$ & $41(17)$ \\
\hline \multicolumn{2}{|l|}{ Size of largest tumor (\%) } \\
\hline $0-3 \mathrm{~cm}$ & $92(39)$ \\
\hline $3-<5 \mathrm{~cm}$ & $75(32)$ \\
\hline $5-<6 \mathrm{~cm}$ & $16(6.7)$ \\
\hline $6-<7 \mathrm{~cm}$ & $15(6.3)$ \\
\hline$\geq 7 \mathrm{~cm}$ & $40(17)$ \\
\hline Within Milan criteria $(\%)^{b}$ & $122(51)$ \\
\hline Beyond Milan criteria (\%) & $116(49)$ \\
\hline Vascular invasion, \% & $38(16)$ \\
\hline Metastasis, \% & $19(8)$ \\
\hline \multicolumn{2}{|l|}{ Treatment of $\mathrm{HCC}^{a}$} \\
\hline Liver transplantation & $0(0.0)$ \\
\hline Surgery (partial hepatectomy) & $5(2.1)$ \\
\hline Systemic chemotherapy (sorafenib) & $69(29)$ \\
\hline Transarterial chemoembolization & $101(42)$ \\
\hline Radiofrequency ablation & $30(13)$ \\
\hline $\mathrm{Y}-90$ radio embolization & $7(2.9)$ \\
\hline Percutaneous ethanol injection & $3(1.3)$ \\
\hline Cryoablation & $1(0.4)$ \\
\hline Other treatment & $11(4.6)$ \\
\hline Any of above treatments & $159(67)$ \\
\hline \multicolumn{2}{|l|}{ HCC contributed to patient's death ${ }^{a}$} \\
\hline Metastatic HCC & $49(21)$ \\
\hline Multifocal HCC (>3 lesions) & $77(32)$ \\
\hline Local or vascular invasion by HCC & $64(27)$ \\
\hline $\begin{array}{l}\text { Large-volume HCC }(>6 \mathrm{~cm} \\
\quad \text { or AFP }>1,000 \mathrm{ng} / \mathrm{mL})\end{array}$ & $107(45)$ \\
\hline Death from complications of $\mathrm{HCC}$ treatment & $6(2.5)$ \\
\hline
\end{tabular}

aThe categories for "method of HCC diagnosis," "treatment of HCC," and "HCC contributed to patient's death" are not mutually exclusive.

${ }^{b}$ Milan criteria: 1 tumor smaller than $5 \mathrm{~cm}$ or 2-3 tumors each of which is smaller than $3 \mathrm{~cm}$.

a histologic diagnosis (Table 2). At the time of diagnosis, $16.0 \%$ had vascular invasion, $8.0 \%$ had metastatic disease, and $51.3 \%$ were within Milan criteria. A large proportion of patients received locoregional treatments, including transarterial chemoembolization in $42.4 \%$ and radiofrequency ablation in $12.7 \%$, whereas $28.4 \%$ were treated with sorafenib and only $2.1 \%$ underwent surgical resection. The criteria that were used to determine that the presence of HCC contributed to the patient's death most commonly included large-volume HCC (45.0\%), multifocal HCC $(32.4 \%)$, local or vascular invasion $(26.9 \%)$, or metastasis (20.6\%). 
Table 3. Distribution of USS and Serum AFP Tests During 0-4 Years Before Index Date

\begin{tabular}{lcc}
\hline & Controls & Cases \\
\hline USS & & \\
All USS tests & 503 & 492 \\
Definitely screening & $287(57.1 \%)$ & $284(57.7 \%)$ \\
Probably screening & $6(1.1 \%)$ & $8(1.6 \%)$ \\
Probably not screening & $4(0.8 \%)$ & $2(0.4 \%)$ \\
Definitely not screening & $206(41.0 \%)$ & $198(40.2 \%)$ \\
AFP & & \\
All AFP tests & 848 & 795 \\
Definitely screening & $641(75.6 \%)$ & $635(79.9 \%)$ \\
Probably screening & $10(1.2 \%)$ & $2(0.3 \%)$ \\
Probably not screening & $0(0.0 \%)$ & $0(0.0 \%)$ \\
Definitely not screening & $197(23.2 \%)$ & $158(19.9 \%)$
\end{tabular}

\section{Association Between Screening and HCC-Related Mortality}

During the 4-year period before the index date, cases underwent 492 USS and 795 serum AFP tests (including 284 and 635, respectively, performed "definitely for screening") and controls underwent a similar number of 503 USS and 848 serum AFP tests (including 287 and 641 , respectively, performed "definitely for screening"; Table 3).

There was no difference between cases and controls in the proportion who underwent screening USS $(52.9 \%$ vs $54.2 \%$, OR 0.95 , 95\% confidence interval 0.66-1.37), screening serum AFP $(74.8 \%$ vs $73.5 \%$, OR $1.07,95 \%$ CI $0.70-1.65)$, or screening USS or AFP $(81.1 \%$ vs $79.4 \%$, OR 1.12 , 95\% CI 0.70-1.81) within 4 years before the index date (Table 4). There also was no difference in receipt of these screening tests within 1,2 , or 3 years before the index date. After adjustment for potential confounders, there was no association between screening with USS or AFP and HCC-related mortality (Table 4).

Receipt of screening with USS and AFP was not associated with HCC-related mortality compared with receipt of USS alone, AFP alone, or no screening at any of the intervals studied (1, 2, 3, or 4 years; Supplemental Table 5).

When we analyzed USS and serum AFP tests that were done definitely or probably for screening (instead of only tests done definitely for screening), we found no association between screening and HCC-related mortality (Supplemental Tables 6 and 7).

\section{Discussion}

HCC screening with USS and/or serum AFP was not associated with decreased risk of HCC-related mortality in this matched case-control study based on recent data from a national health care system in the United States.

Consensus on HCC screening recommendations is lacking among professional societies. Most liver societies such as the AASLD, ${ }^{2}$ the European Association for the Study of the Liver, ${ }^{3}$ and the Asian Pacific Association for the Study of the
Liver $^{4}$ have recommended USS every 6 months with or without concomitant serum AFP for HCC surveillance in patients with cirrhosis. In contrast, non-liver societies have not endorsed HCC screening because of the lack of highquality data. The US Preventive Services Task Force has not adopted an HCC practice guideline, the American Cancer Society makes no recommendation on HCC screening, ${ }^{5}$ and the National Cancer Institute found no evidence that screening decreases mortality from HCC but did find evidence that screening could result in harm. ${ }^{6}$

It remains unclear whether HCC screening decreases HCC-related mortality. Although 2 RCTs were performed in patients with hepatitis B virus infection in China before 1997, their results do not apply to patients with cirrhosis in the current era in the United States and have been criticized for methodologic limitations. ${ }^{9}$ The remaining studies were observational, including 39 aggregated in 2 metaanalyses $^{9,10}$ and 1 large VA study published subsequently. ${ }^{43}$ However, these studies did not compare HCC-related mortality in screened vs unscreened patients. Rather, they compared survival after the diagnosis of HCC in patients whose HCC was diagnosed because of screening vs those in whom HCC presented with symptoms. This study design is inherently susceptible to lead-time bias that can lead to overestimation of the benefits of screening. Several studies have attempted to adjust for lead-time bias by estimating the "sojourn time" ${ }^{44}$ (the period during which the tumor is asymptomatic but detectable by screening) using estimates of tumor growth rate or doubling time. The conclusions of these studies vary dramatically depending on the estimates of tumor doubling time and sojourn time used to adjust for lead-time bias. ${ }^{45,46}$ In addition, this study design is limited by length-time bias (aggressive tumors are more likely to present symptomatically and less likely to be diagnosed by screening than less aggressive tumors) and by selection bias (patients who underwent screening were a selected subset of all patients with cirrhosis who might have had improved survival by having access to better overall care). Moreover, most of these studies did not adjust for MELD score, a critical determinant of survival in patients with HCC.

Our methodology addresses many of the limitations of prior studies examining HCC screening. We used a matched case-control study design to evaluate HCC screening effectiveness that is not susceptible to lead-time or length-time bias. Because cases are defined as patients with cirrhosis and fatal HCC, this study design yields estimates of the impact of screening on HCC-related mortality and approximates the results that would be expected from a randomized controlled trial. ${ }^{15}$ We evaluated a large number of cases and matched controls who were derived randomly from a national health care system that provides care to 8 million veterans in 180 medical centers across the entire United States; thus, our findings are typical of community-based settings. All VA patients have uniform access to medical care, limiting bias owing to differential access to HCC screening and HCC treatments. Potential cases and controls were individually verified by chart review using 
Table 4. Comparison of Cases and Controls for Occurrence of Screening ${ }^{a}$ USS, Screening AFP, or USS or AFP at Given Intervals Before Index Date

\begin{tabular}{|c|c|c|c|c|}
\hline & $\begin{array}{c}\text { Controls } \\
(\mathrm{n}=238), \mathrm{n}(\%)\end{array}$ & $\begin{array}{c}\text { Cases } \\
(\mathrm{n}=238), \mathrm{n}(\%)\end{array}$ & $\begin{array}{l}\text { Odds ratio } \\
(95 \% \mathrm{Cl})\end{array}$ & $\begin{array}{l}\text { Adjusted }{ }^{C} \text { Odds } \\
\text { ratio }(95 \% \mathrm{Cl})\end{array}$ \\
\hline \multicolumn{5}{|c|}{$0-4$ y before index date } \\
\hline AFP & 175 (73.5) & $178(74.8)$ & 1.07 (0.70-1.65) & $1.08(0.67-1.75)$ \\
\hline USS or AFP & 189 (79.4) & $193(81.1)$ & $1.12(0.70-1.81)$ & $1.11(0.68-1.82)$ \\
\hline \multicolumn{5}{|c|}{$0-3$ y before index date } \\
\hline USS or AFP & $177(74.4)$ & $182(76.5)$ & $1.13(0.73-1.74)$ & $1.14(0.72-1.79)$ \\
\hline \multicolumn{5}{|c|}{$0-2$ y before index date } \\
\hline USS & 95 (39.9) & $91(38.2)$ & $0.93(0.63-1.36)$ & $0.93(0.60-1.43)$ \\
\hline AFP & $145(60.9)$ & $151(63.4)$ & $1.13(0.76-1.69)$ & $1.18(0.76-1.83)$ \\
\hline USS or AFP & $160(67.2)$ & $165(69.3)$ & $1.12(0.74-1.68)$ & $1.12(0.73-1.73)$ \\
\hline \multicolumn{5}{|c|}{$0-1$ y before index date } \\
\hline
\end{tabular}

\begin{abstract}
aOnly tests performed "definitely for screening" were included in this analysis.
${ }^{b} \mathrm{OR}$ of screening in cases relative to controls. An OR less than 1 would be indicative of an association between HCC surveillance and decreased HCC-related mortality. Although this OR is unadjusted, cases and controls were matched for age, sex, race, etiology of cirrhosis, MELD score at time of cirrhosis diagnosis, date of cirrhosis diagnosis, and VA facility.

${ }^{c}$ Adjusted for age, etiology of cirrhosis, MELD score at cirrhosis diagnosis, race and ethnicity, year of cirrhosis diagnosis, diabetes, alcohol use disorders, body mass index, eradication of HCV by antiviral treatment, and receipt of abdominal CT or MRI during the period of interest. Also, USS analysis was adjusted for screening for serum AFP and serum AFP analysis was adjusted for screening by USS.
\end{abstract}

prespecified criteria. Cases were limited to patients who died within a very recent period (2013-2015), such that current treatments for HCC would be available to potentially affect HCC-related mortality. Controls were matched to cases for important characteristics that affect receipt of screening or death from HCC, and additional potential confounders were adjusted for. Blinding to the case or control status of patients was maintained for the analyst who identified all USS and serum AFP tests performed in the 4 years before the index date, the research assistant who copied relevant reports from the electronic medical records onto our REDCap database, and the physicianinvestigator who determined whether USS or serum AFP tests were done for screening. The medical records related to each of the 995 USS and 1643 AFP tests performed in the 4-year period before the index date were reviewed by a physician-investigator to determine whether the USS or AFP test was performed for screening. We excluded cases and controls who had a MELD score of at least 20 before the index date, because screening is not recommended in patients with decompensated cirrhosis (unless they are listed for liver transplantation) because they are unlikely to benefit from HCC treatment.

Our study had several potential limitations. It was conducted in a male population of US veterans, which might limit the generalizability of our study to women. However, we are not aware of studies showing that the test characteristics of screening USS or serum AFP or the outcomes of HCC treatment are different in men vs women with cirrhosis. Nonetheless, it would be useful to replicate our case-control study in a different health care system whose records would allow the accurate identification of the indication for USS and AFP testing because, to our knowledge, this is the only available case-control study of HCC screening effectiveness. The main limitation of case-control studies of cancer screening effectiveness is the potential for misclassifying as screening tests some tests that were actually done to evaluate symptoms or signs of cancer in the cases. In our study, some USS or serum AFP tests might have been performed in cases to evaluate suspected HCC, with the basis for the suspicion not specifically mentioned in the medical record. We would have misclassified these as "screening" tests, leading to falsely high ORs and thus a falsely low estimate of screening effectiveness. This is less a concern for USS, for which the indication has to be recorded in the report (which was available to us), but potentially more of a concern for serum AFP tests, for which the ordering provider's progress notes were the main source of information on test indication.

Two conditions are necessary for HCC screening to result in a decrease in HCC-related mortality. First, screening USS or serum AFP must be able to detect HCC at an earlier stage than it would otherwise present as a result of symptoms, signs, or incidental imaging. Second, treatment must be available for this early-stage disease that yields superior outcomes relative to treatment of disease detected in the absence of screening. The lack of effectiveness of HCC screening in our study could be related to failure in one or 
both of these conditions. Multiple studies suggest that HCCs detected by screening USS or serum AFP have, on average, an earlier stage at diagnosis than HCCs detected by symp-toms, signs, or incidental imaging. ${ }^{33,46-49}$ However, this does not prove that screening leads to earlier detection. Another explanation is that screening is more likely to identify slowgrowing tumors, which have a lower stage, and more likely to miss the fast-growing tumors, which are identified at a higher stage by symptoms. It is possible that the HCCs most likely to lead to death are the HCCs least likely to be identified by current screening modalities at an early stage. Whether early treatment for HCC in patients with cirrhosis leads to a decrease in case fatality is questionable. Patients who receive locoregional treatments or surgical resection remain at risk of developing recurrent HCC, new HCC, and progressive liver dysfunction from their underlying cirrhosis. Liver transplantation is the only treatment that can cure the cancer and the underlying cirrhosis and should confer a survival benefit. However, only a small minority of patients with HCC undergo liver transplantation. In 2012, 24,696 incident cases of HCC were reported in the US Cancer Statistics registry ${ }^{50}$ (which, if anything, un-derestimates the total number of HCC cases), whereas only 1,733 (7\%) liver transplantations were performed for HCC in the United States. ${ }^{51}$ Pragmatic RCTs currently under way, which randomize patients to HCC surveillance outreach with patient education and patient navigation services vs "opportunistic" surveillance, ${ }^{52}$ could address the impact of surveillance of early detection and receipt of treatment but are not designed to study cancer-related mortality.

It is unlikely that the lack of screening-related survival benefit in our study was due to untimely diagnostic and confirmatory tests for HCC or unavailability of HCC treatments in the VA system. First, even among these fatal cases, $51.3 \%$ were diagnosed within Milan criteria, a much larger proportion than that of unselected patients with HCC in the national SEER registry diagnosed within Milan criteria (36.4\% in 2003-2006 and 46.3\% in 2013-2014)..$^{53}$ Second, we found that even among these fatal cases, who had very advanced HCC at presentation, a substantial proportion $(66.8 \%)$ received a cancer-specific treatment. The fact that none of the cases received liver transplantation is not indicative of unavailability of liver transplantation, but rather a result of the fact that only carefully selected patients who are not expected to die of HCC undergo liver transplantation and, hence, liver transplant recipients did not contribute to the fatal cases in our study. It is unlikely that the lack of screening-related survival benefit was due to patients having advanced cirrhosis, which could discourage screening, preclude certain HCC treatments, or dictate patient survival irrespective of the presence of HCC, because patients had a MELD score lower than 20 at all times before the index date and a mean MELD score of 9 at the time of cirrhosis diagnosis.

In summary, we found no evidence that screening with USS or serum AFP decreases HCC-related mortality in patients with cirrhosis. This suggests that these screening tests and/or the currently available treatments are suboptimal and need to be improved.

\section{Supplementary Material}

Note: To access the supplementary material accompanying this article, visit the online version of Gastroenterology at www.gastrojournal.org, and at https://doi.org/10.1053/ j.gastro.2018.06.079.

\section{References}

1. El-Serag HB. Hepatocellular carcinoma. N Engl J Med 2011;365:1118-1127.

2. Heimbach J, Kulik LM, Finn R, et al. AASLD guidelines for the treatment of hepatocellular carcinoma. Hepatology 2018;67:358-380.

3. European Association for the Study of the Liver and European Organisation for Research Treatment of Cancer (EASL-EORTC) clinical practice guidelines: management of hepatocellular carcinoma. J Hepatol 2012;56:908-943.

4. Omata M, Cheng AL, Kokudo N, et al. Asia-Pacific clinical practice guidelines on the management of hepatocellular carcinoma: a 2017 update. Hepatol Int 2017; 11:317-370.

5. American Cancer Society. Can Liver Cancer Be Found Early? Volume 2018. Atlanta, GA: American Cancer Society, 2016.

6. National Cancer Institute. Liver (hepatocellular) cancer screening - health professional version. Available at: https://www.cancer.gov/types/liver/hp/liver-screeningpdq. Accessed January 9, 2018.

7. Zhang BH, Yang BH, Tang ZY. Randomized controlled trial of screening for hepatocellular carcinoma. J Cancer Res Clin Oncol 2004;130:417-422.

8. Chen JG, Parkin DM, Chen QG, et al. Screening for liver cancer: results of a randomised controlled trial in Qidong, China. J Med Screen 2003;10:204-209.

9. Kansagara D, Papak J, Pasha AS, et al. Screening for hepatocellular carcinoma in chronic liver disease: a systematic review. Ann Intern Med 2014;161:261-269.

10. Singal AG, Pillai A, Tiro J. Early detection, curative treatment, and survival rates for hepatocellular carcinoma surveillance in patients with cirrhosis: a metaanalysis. PLoS Med 2014;11:e1001624.

11. Bruix J, Sherman M. Management of hepatocellular carcinoma: an update. Hepatology 2011;53:1020-1022.

12. Poustchi H, Farrell GC, Strasser SI, et al. Feasibility of conducting a randomized control trial for liver cancer screening: is a randomized controlled trial for liver cancer screening feasible or still needed? Hepatology 2011; 54:1998-2004.

13. Lederle FA, Pocha C. Screening for liver cancer: the rush to judgment. Ann Intern Med 2012;156:387-389.

14. Kelley MJ. Surveillance for hepatocellular carcinoma. Ann Intern Med 2011;155:274; author reply 275.

15. Morrison AS. Screening in Chronic Disease. New York: Oxford University Press; 1992.

16. Weiss NS, Etzioni R. Estimating the influence of rescreening interval on the benefits associated with cancer screening: approaches and limitations. Epidemiology 2002;13:713-717. 
17. Weiss NS, Dhillon PK, Etzioni R. Case-control studies of the efficacy of cancer screening: overcoming bias from nonrandom patterns of screening. Epidemiology 2004; 15:409-413.

18. Selby JV, Friedman GD, Quesenberry CP Jr, et al. Effect of fecal occult blood testing on mortality from colorectal cancer. A case-control study. Ann Intern Med 1993; 118:1-6.

19. Selby JV, Friedman GD, Quesenberry CP Jr, et al. A case-control study of screening sigmoidoscopy and mortality from colorectal cancer. N Engl J Med 1992; 326:653-657.

20. Newcomb PA, Weiss NS, Storer BE, et al. Breast selfexamination in relation to the occurrence of advanced breast cancer. J Natl Cancer Inst 1991;83:260-265.

21. Corley DA, Mehtani K, Quesenberry C, et al. Impact of endoscopic surveillance on mortality from Barrett's esophagus-associated esophageal adenocarcinomas. Gastroenterology 2013;145:312-319 e1.

22. Rustagi AS, Kamineni A, Weinmann S, et al. Cervical screening and cervical cancer death among older women: a population-based, case-control study. Am J Epidemiol 2014;179:1107-1114.

23. Friedman GD, Hiatt RA, Quesenberry CP Jr, et al. Casecontrol study of screening for prostatic cancer by digital rectal examinations. Lancet 1991;337:1526-1529.

24. Berwick M, Begg CB, Fine JA, et al. Screening for cutaneous melanoma by skin self-examination. J Natl Cancer Inst 1996;88:17-23.

25. Veterans Affairs Corporate Data Warehouse. Available at: http://www.hsrd.research.va.gov/for_researchers/vinci/ cdw.cfm. Accessed December 19, 2016.

26. Harris PA, Taylor R, Thielke R, et al. Research electronic data capture (REDCap)—a metadata-driven methodology and workflow process for providing translational research informatics support. J Biomed Inform 2009; 42:377-381.

27. Kramer JR, Davila JA, Miller ED, et al. The validity of viral hepatitis and chronic liver disease diagnoses in Veterans Affairs administrative databases. Aliment Pharmacol Ther 2008;27:274-282.

28. Kramer JR, Giordano TP, Souchek J, et al. The effect of HIV coinfection on the risk of cirrhosis and hepatocellular carcinoma in U.S. veterans with hepatitis C. Am J Gastroenterol 2005;100:56-63.

29. Ioannou GN, Splan MF, Weiss NS, et al. Incidence and predictors of hepatocellular carcinoma in patients with cirrhosis. Clin Gastroenterol Hepatol 2007; 5:938-945.

30. Ioannou GN, Bryson CL, Weiss NS, et al. The prevalence of cirrhosis and hepatocellular carcinoma in patients with human immunodeficiency virus infection. Hepatology 2013;57:249-257.

31. Ioannou GN, Beste LA, Green PK. Similar effectiveness of boceprevir and telaprevir treatment regimens for hepatitis C virus infection, based on a nationwide study of veterans. Clin Gastroenterol Hepatol 2014; 12:1371-1380.

32. Ioannou GN, Scott JD, Yang Y, et al. Rates and predictors of response to anti-viral treatment for hepatitis $\mathrm{C}$ virus in HIV/HCV co-infection in a nationwide study of 619 patients. Aliment Pharmacol Ther 2013; 38:1373-1384.

33. Davila JA, Henderson L, Kramer JR, et al. Utilization of surveillance for hepatocellular carcinoma among hepatitis C virus-infected veterans in the United States. Ann Intern Med 2011;154:85-93.

34. El-Serag HB, Johnson ML, Hachem C, et al. Statins are associated with a reduced risk of hepatocellular carcinoma in a large cohort of patients with diabetes. Gastroenterology 2009;136:1601-1608.

35. Kanwal F, Hoang T, Kramer JR, et al. Increasing prevalence of HCC and cirrhosis in patients with chronic hepatitis C virus infection. Gastroenterology 2011; 140:1182-1188 e1.

36. Davila JA, Kramer JR, Duan Z, et al. Referral and receipt of treatment for hepatocellular carcinoma in United States veterans: effect of patient and nonpatient factors. Hepatology 2013;57:1858-1868.

37. El-Serag HB, Kanwal F, Davila JA, et al. A new laboratory-based algorithm to predict development of hepatocellular carcinoma in patients with hepatitis $\mathrm{C}$ and cirrhosis. Gastroenterology 2014;146:1249-1255.e1.

38. Mitchell DG, Bruix J, Sherman M, et al. LI-RADS (Liver Imaging Reporting and Data System): summary, discussion, and consensus of the LI-RADS Management Working Group and future directions. Hepatology 2015; 61:1056-1065.

39. Beste LA, Leipertz SL, Green PK, et al. Trends in burden of cirrhosis and hepatocellular carcinoma by underlying liver disease in US veterans, 2001-2013. Gastroenterology 2015;149:1471-1482 e5.

40. Sheu JC, Sung JL, Chen DS, et al. Growth rate of asymptomatic hepatocellular carcinoma and its clinical implications. Gastroenterology 1985;89:259-266.

41. Etzioni RD, Weiss NS. Analysis of case-control studies of screening: impact of misspecifying the duration of detectable preclinical pathologic changes. Am J Epidemiol 1998;148:292-297.

42. Dupont WD. Power calculations for matched casecontrol studies. Biometrics 1988;44:1157-1168.

43. Mittal S, Kanwal F, Ying J, et al. Effectiveness of surveillance for hepatocellular carcinoma in clinical practice: a United States cohort. J Hepatol 2016; 65:1148-1154.

44. Duffy SW, Nagtegaal ID, Wallis M, et al. Correcting for lead time and length bias in estimating the effect of screen detection on cancer survival. Am J Epidemiol 2008;168:98-104.

45. Cucchetti A, Trevisani F, Pecorelli A, et al. Estimation of lead-time bias and its impact on the outcome of surveillance for the early diagnosis of hepatocellular carcinoma. J Hepatol 2014;61:333-341.

46. El-Serag HB, Kramer JR, Chen GJ, et al. Effectiveness of AFP and ultrasound tests on hepatocellular carcinoma mortality in HCV-infected patients in the USA. Gut 2011; 60:992-997.

47. Giannini E, Arzani L, Borro P, et al. Does surveillance for hepatocellular carcinoma in $\mathrm{HCV}$ cirrhotic patients improve treatment outcome mainly due to better clinical 
status at diagnosis? Hepatogastroenterology 2000; 47:1395-1398.

48. Kemp W, Pianko S, Nguyen S, et al. Survival in hepatocellular carcinoma: impact of screening and etiology of liver disease. J Gastroenterol Hepatol 2005;20:873-881.

49. Leykum LK, El-Serag HB, Cornell J, et al. Screening for hepatocellular carcinoma among veterans with hepatitis $\mathrm{C}$ on disease stage, treatment received, and survival. Clin Gastroenterol Hepatol 2007;5:508-512.

50. White DL, Thrift AP, Kanwal F, et al. Incidence of hepatocellular carcinoma in all 50 United States, from 2000 through 2012. Gastroenterology 2017;152:812-820 e5.

51. US Department of Health and Human Services. Organ Procurement and Transplantation Network. National data. Available at: https://optn.transplant.hrsa.gov/data/viewdata-reports/national-data/. Accessed February 13, 2018.

52. Texas Hepatocellular Carcinoma Consortium (THCCC) Project 5. ClinicalTrials.gov Identifier: NCT02582918. Available at: https://clinicaltrials.gov/ct2/show/NCT02582918? term $=$ Texas + Hepatocellular + Carcinoma + Consortium \& rank=1. Accessed May 6, 2018.

53. Robinson A, Tavakoli H, Liu B, et al. Advanced hepatocellular carcinoma tumor stage at diagnosis in the 19451965 birth cohort reflects poor use of hepatocellular carcinoma screening. Hepatol Commun 2018. Available at: https://aasldpubs.onlinelibrary.wiley.com/doi/pdf/ 10.1002/hep4.1236. Accessed September 1, 2018.
Received March 12, 2018. Accepted June 26, 2018.

\section{Reprint requests}

Address requests for reprints to: George loannou, BMBCh, MS, Veterans Affairs Puget Sound Health Care System, Gastroenterology, S-111-Gastro, 1660 S Columbian Way, Seattle, WA 98108. e-mail: georgei@medicine.washington.edu.

\section{Acknowledgments}

Author contributions: All authors approved the final version of the manuscript. $\mathrm{Gl}$ is the guarantor of this paper. AM was responsible for the abstraction of medical charts, study design, statistical analysis and interpretation of data, drafting of the manuscript, and critical revision of the manuscript. FS was responsible for the study design and critical revision of the manuscript. G-YJ created the REDCap database and accessed electronic medical records. LB was responsible for the study concept and design, acquisition of data, statistical analysis and interpretation of data, and critical revision of the manuscript. NW was responsible for the study concept and design, statistical analysis and interpretation of data, drafting of the manuscript, critical revision of the manuscript, and obtaining funding. $\mathrm{SH}$ was responsible for the study concept and design, interpretation of the data, and critical revision of the manuscript. EL was responsible for acquisition of data and statistical analysis and interpretation of data. KB was responsible for the study design, analysis of data, interpretation of data, and critical revision of manuscript. Gl was responsible for the study concept and design, acquisition of data, abstraction of medical charts, statistical analysis and interpretation of data, drafting of the manuscript, critical revision of the manuscript, and obtaining funding.

\section{Conflicts of interest}

Authors disclose no conflicts.

Funding

$\mathrm{NIH} / \mathrm{NCl}$ grant R01CA196692 and VA CSR\&D grant I01CX001156 to GNI and NSW. The funding source played no role in the study design or collection, analysis, or interpretation of data. The contents do not represent the views of the US Department of Veterans Affairs or the United States Government. 
Supplemental Table 1.Definition of Patient Characteristics Based on Diagnostic ICD-9 Codes Recorded at Least Twice in Inpatient or Outpatient Records

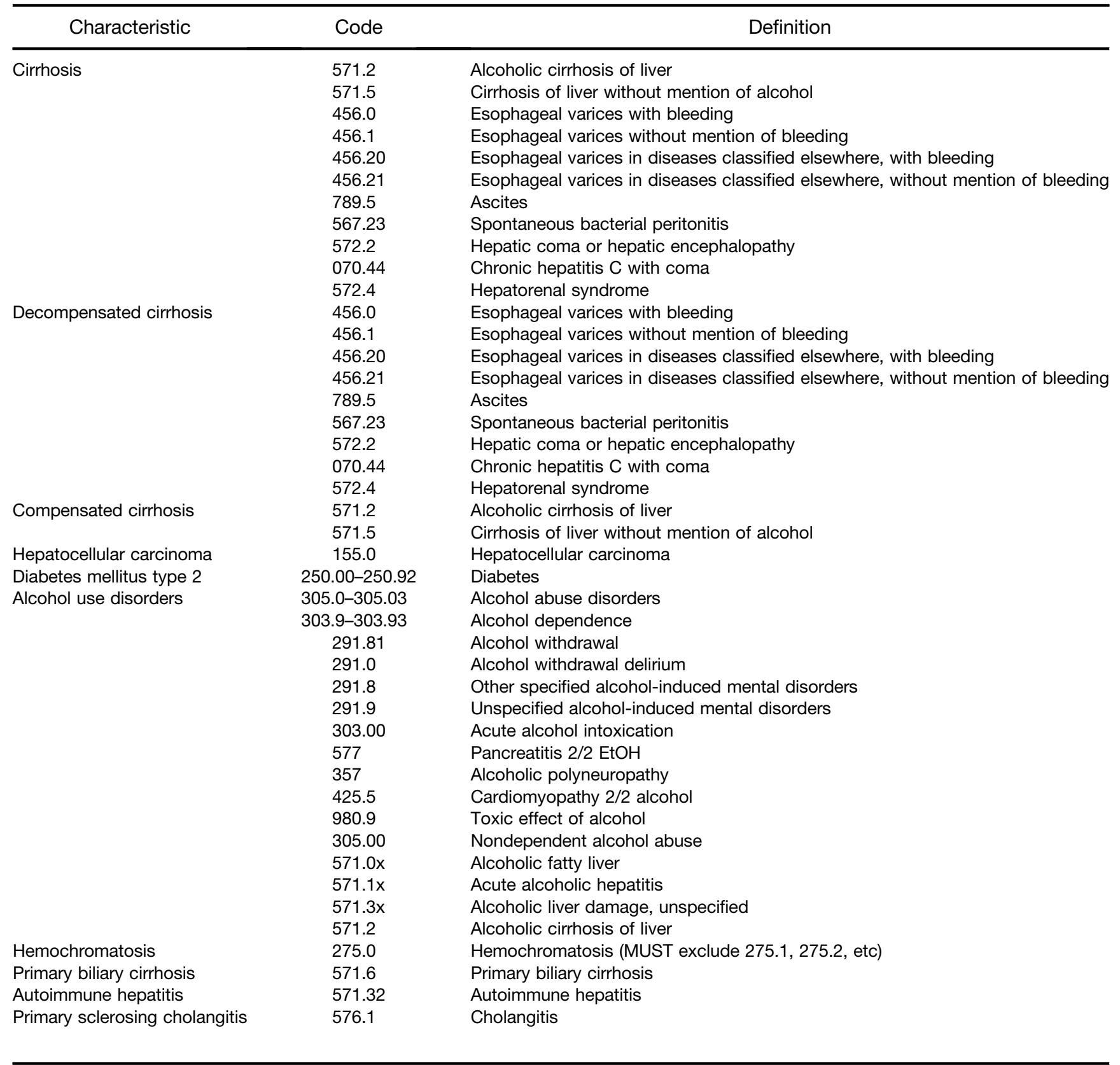


Supplemental Table 2. Definitions of Categories for Etiology of Cirrhosis ${ }^{34}$

\begin{tabular}{|c|c|}
\hline Etiology of cirrhosis ${ }^{a}$ & Definition \\
\hline Hepatitis $\mathrm{C}$ virus (HCV) & $\begin{array}{l}\text { Patients with a positive serum HCV RNA were categorized as having HCV regardless of any additional } \\
\text { etiologies. }\end{array}$ \\
\hline Alcoholic liver disease (ALD) & $\begin{array}{l}\text { Patients with } I C D-9 \text { codes for alcohol use disorders in the absence of serologic markers of chronic HCV or } \\
\text { hepatitis B virus infection and in the absence of } I C D-9 \text { codes for hemochromatosis, primary biliary } \\
\text { cirrhosis, primary sclerosing cholangitis, and autoimmune hepatitis. }\end{array}$ \\
\hline $\begin{array}{l}\text { Nonalcoholic fatty liver } \\
\text { disease (NAFLD) }\end{array}$ & $\begin{array}{l}\text { Patients with diabetes (ICD-9 codes } 250-250.92 \text {, recorded at least twice) or body mass index } \geq 30 \mathrm{~kg} / \mathrm{m}^{2} \\
\text { before diagnosis of cirrhosis who did not have HCV, HBV, ALD (defined as above) or ICD-9 codes for } \\
\text { hemochromatosis, primary biliary cirrhosis, primary sclerosing cholangitis, and autoimmune hepatitis. } \\
\text { NAFLD-related cirrhosis does not have pathognomonic serologic, radiologic, or histologic features-even } \\
\text { hepatic steatosis is frequently absent after cirrhosis develops. Hence, we adapted a clinical definition of } \\
\text { NAFLD based on previous work that reflects the diagnostic process used in clinical practice, in which } \\
\text { NAFLD is suspected in the presence of risk factors such as obesity and diabetes after exclusion of other } \\
\text { etiologies. }\end{array}$ \\
\hline
\end{tabular}

Other

All other patients not meeting criteria above for HCV, ALD, or NAFLD.

aThese definitions were necessarily designed to be mutually exclusive.

Supplemental Table 3. Determination of Whether Abdominal USS Was Done for Screening or Non-Screening Indications

Indication reported in ultrasound report or in the ordering provider progress notes before and after Assignment of indication the USS was ordered and performed.

a. Definitely screening ${ }^{a}$

HCC (or hepatoma or liver cancer) screening or surveillance in patients with no new symptoms, signs, or test results suggestive of HCC.

Indications completely unrelated to liver disease such as abdominal aortic aneurysm screening.

Follow-up renal cysts, etc.

b. Definitely non-screening

As workup of any symptoms or signs that might be suggestive of HCC including increased (or worsening) liver function test results; new ascites; weight loss; abdominal pain; abdominal tenderness; abdominal mass; abdominal distention; abdominal bloating; enlarged liver; palpable liver; failure to thrive; jaundice; increased bilirubin; variceal bleeding; or fever.

As a follow-up of another positive test result suggestive of HCC: abnormal AFP; liver abnormality on abdominal ultrasound; liver abnormality on abdominal CT or MRI scan.

Any test done in the emergency room or as an inpatient.

c. Probably screening

Screen for HCC and patient also has ascites or encephalopathy (when ascites or encephalopathy are longstanding or unchanged). $b$

d. Probably non-screening Unclear if patient has symptoms or signs suspicious of HCC.

anly record as "definitely screening" if there is no simultaneous "non-screening" indication.

${ }^{b}$ This refers to tests in which the provider seems to be ordering the USS with the intention of screening for HCC but also wants a comment on the degree of ascites that is long-standing and being treated, without any obvious concern that development of HCC might have contributed to the ascites or encephalopathy. 
Supplemental Table 4. Determination of Whether a Serum AFP Test Was Done for Screening or Non-Screening Indications

Assignment of indication

Indication reported in the ordering provider progress notes before and after serum AFP test was

a. Definitely screening ${ }^{a}$

HCC (or hepatoma or liver cancer) screening or surveillance in patients with no new symptoms, signs, or test results suggestive of HCC.

b. Definitely non-screening

As workup of any symptoms or signs that might be suggestive of HCC including increased (or worsening) liver function test results; new ascites; weight loss; abdominal pain; abdominal tenderness; abdominal mass; abdominal distension; abdominal bloating; enlarged liver; palpable liver; failure to thrive; jaundice; increased bilirubin; variceal bleeding; or fever.

As a follow-up of another positive test result suggestive of HCC: abnormal AFP; liver abnormality on abdominal ultrasound; liver abnormality on abdominal CT or MRI scan.

c. Probably screening

Any test done in the emergency room or as an inpatient.

Screen for HCC and patient also has ascites or encephalopathy (when ascites or encephalopathy are longstanding or unchanged). ${ }^{b}$

d. Probably non-screening

Rule out HCC, but unclear based on documentation if patient has symptoms or signs suspicious for HCC.

"Only record as "definitely screening" if there is no simultaneous "non-screening" indication.

${ }^{b}$ This refers to serum AFP tests ordered in patients who have ascites or encephalopathy that are long-standing and being treated, without any obvious concern that development of HCC might have contributed to the ascites or encephalopathy. 
Supplemental Table 5. Comparison of Cases and Controls for Occurrence of Screening for HCC With Serum AFP and USS vs Only 1 of the 2 Tests or None

\begin{tabular}{|c|c|c|c|c|c|c|c|c|}
\hline & $\begin{array}{c}\text { Controls } \\
(n=238), n(\%)\end{array}$ & $\begin{array}{c}\text { Cases } \\
(\mathrm{n}=238), \mathrm{n}(\%)\end{array}$ & $\begin{array}{l}\text { OR }(95 \% \mathrm{Cl}) \\
\text { AFP + USS vs } \\
\text { USS only }\end{array}$ & $\begin{array}{c}\text { Adjusted }^{a} \text { OR } \\
\text { (95\% Cl), AFP + } \\
\text { USS vs USS only }\end{array}$ & $\begin{array}{l}\text { OR }(95 \% \mathrm{Cl}), \\
\text { AFP + USS vs } \\
\text { AFP only }\end{array}$ & $\begin{array}{l}\text { Adjusted }{ }^{a} \text { OR } \\
\text { (95\% CI), AFP + } \\
\text { USS vs AFP only }\end{array}$ & $\begin{array}{l}\text { OR }(95 \% \mathrm{Cl}) \\
\text { AFP + USS } \\
\text { vs neither }\end{array}$ & $\begin{array}{l}\text { Adjusted }^{a} \text { OR } \\
\text { (95\% CI), AFP + } \\
\text { USS vs neither }\end{array}$ \\
\hline \multicolumn{9}{|c|}{$0-4$ y before index date } \\
\hline None & 49 (20.6) & 45 (18.9) & & & & & 1 & 1 \\
\hline USS only & $14(5.9)$ & 15 (6.3) & & & 1 & 1 & & \\
\hline AFP only & $60(25.2)$ & 67 (28.2) & 1 & 1 & & & & \\
\hline USS + AFP & 115 (48.3) & $111(46.6)$ & $0.86(0.29-2.55)$ & $1.35(0.38-4.82)$ & $0.97(0.58-1.61)$ & $1.09(0.63-1.88)$ & $0.90(0.49-1.68)$ & $0.87(0.44-1.72)$ \\
\hline \multicolumn{9}{|c|}{$0-3$ y before index date } \\
\hline None & $61(25.6)$ & $56(23.5)$ & & & & & 1 & 1 \\
\hline USS only & $13(5.5)$ & $14(5.9)$ & & & 1 & 1 & & \\
\hline AFP only & $60(25.2)$ & $70(29.4)$ & 1 & 1 & & & & \\
\hline USS + AFP & $104(43.7)$ & 98 (41.2) & $0.86(0.29-2.55)$ & $1.23(0.35-4.33)$ & $0.75(0.44-1.27)$ & $0.84(0.48-1.48)$ & $1.15(0.63-2.09)$ & $1.21(0.63-2.33)$ \\
\hline \multicolumn{9}{|c|}{$0-2$ y before index date } \\
\hline None & 78 (32.8) & $73(30.7)$ & & & & & 1 & 1 \\
\hline USS only & $15(6.3)$ & $14(5.9)$ & & & 1 & 1 & & \\
\hline AFP only & 65 (27.3) & 74 (31.1) & 1 & 1 & & & & \\
\hline USS + AFP & $80(33.6)$ & 77 (32.4) & $1.67(0.40-6.97)$ & $2.07(0.44-9.66)$ & $0.63(0.34-1.16)$ & $0.79(0.41-1.53)$ & $1.28(0.69-2.37)$ & $1.33(0.69-2.57)$ \\
\hline \multicolumn{9}{|c|}{$0-1$ y before index date } \\
\hline None & $111(46.6)$ & 95 (39.9) & & & & & 1 & 1 \\
\hline USS only & $18(7.6)$ & $22(9.2)$ & & & 1 & 1 & & \\
\hline AFP only & 65 (27.3) & $73(30.7)$ & 1 & 1 & & & & \\
\hline USS + AFP & $44(18.5)$ & $48(20.2)$ & $0.60(0.14-2.51)$ & $0.65(0.12-3.62)$ & $0.87(0.41-1.82)$ & $0.97(0.44-2.16)$ & $1.67(0.81-3.41)$ & $1.57(0.73-3.34)$ \\
\hline
\end{tabular}

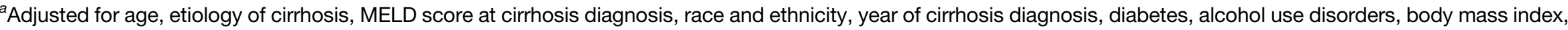
receipt of abdominal CT or MRI during the period of interest, and eradication of HCV by antiviral treatment. 
Supplemental Table 6. Comparison of Cases and Controls for Occurrence of Definite or Probable Screening Ultrasound, Screening AFP, or USS or AFP at Given Intervals Before Index Date

\begin{tabular}{|c|c|c|c|c|}
\hline & Controls $(n=238), n(\%)$ & Cases $(n=238), n(\%)$ & OR $(95 \% \mathrm{Cl})$ & Adjusted $^{a}$ OR $(95 \% \mathrm{Cl})$ \\
\hline \multicolumn{5}{|c|}{$0-4$ y before index date } \\
\hline USS & $131(55.0)$ & $128(53.8)$ & $0.95(0.66-1.37)$ & $0.94(0.63-1.41)$ \\
\hline USS or AFP & $190(79.8)$ & $193(81.1)$ & $1.09(0.68-1.75)$ & $1.07(0.66-1.75)$ \\
\hline \multicolumn{5}{|c|}{$0-3$ y before index date } \\
\hline USS & $119(50.0)$ & $113(47.5)$ & $0.90(0.63-1.30)$ & $0.89(0.59-1.34)$ \\
\hline \multicolumn{5}{|c|}{$0-2$ y before index date } \\
\hline USS & $97(40.8)$ & $91(38.2)$ & $0.89(0.61-1.31)$ & $0.88(0.57-1.35)$ \\
\hline AFP & $146(61.3)$ & $152(63.9)$ & $1.13(0.76-1.69)$ & $1.22(0.78-1.91)$ \\
\hline USS or AFP & $161(67.6)$ & $166(69.7)$ & $1.12(0.74-1.68)$ & $1.13(0.73-1.74)$ \\
\hline \multicolumn{5}{|c|}{$0-1$ y before index date } \\
\hline USS & $65(27.3)$ & $70(29.4)$ & $1.11(0.74-1.67)$ & $1.08(0.70-1.68)$ \\
\hline
\end{tabular}

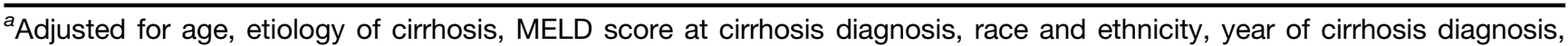
diabetes, alcohol use disorders, body mass index, receipt of abdominal CT or MRI during the period of interest, and eradication of HCV by antiviral treatment. Also, USS analysis was adjusted for serum AFP and serum AFP analysis was adjusted for USS. 


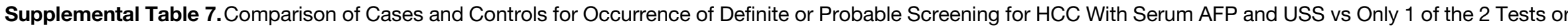
None

\begin{tabular}{|c|c|c|c|c|c|c|c|c|}
\hline & $\begin{array}{c}\text { Controls } \\
(n=238), n(\%)\end{array}$ & $\begin{array}{c}\text { Cases } \\
(n=238), n(\%)\end{array}$ & $\begin{array}{l}\text { OR (95\% Cl), } \\
\text { AFP + USS vs } \\
\text { USS only }\end{array}$ & $\begin{array}{c}\text { Adjusted }^{a} \text { OR } \\
(95 \% \mathrm{Cl}) \text {, AFP + } \\
\text { USS vs USS only }\end{array}$ & $\begin{array}{c}\text { OR }(95 \% \mathrm{Cl}) \\
\text { AFP + USS vs } \\
\text { AFP only }\end{array}$ & $\begin{array}{l}\text { Adjusted }^{a} \text { OR } \\
(95 \% \mathrm{Cl}), \text { AFP }+ \\
\text { USS vs AFP only }\end{array}$ & $\begin{array}{l}\text { OR }(95 \% \mathrm{Cl}) \\
\text { AFP + USS } \\
\text { vs none }\end{array}$ & $\begin{array}{c}\text { Adjusted }^{a} \text { OR } \\
(95 \% \text { Cl), AFP + } \\
\text { USS vs none }\end{array}$ \\
\hline \multicolumn{9}{|c|}{$0-4$ y before index date } \\
\hline None & $48(20.2)$ & 45 (18.9) & & & & & 1 & 1 \\
\hline USS only & $15(6.3)$ & $15(6.3)$ & & & 1 & 1 & & \\
\hline AFP only & $59(24.8)$ & 65 (27.3) & 1 & 1 & & & & \\
\hline USS + AFP & $116(48.7)$ & $113(47.5)$ & $0.86(0.29-2.55)$ & $1.37(0.38-4.93)$ & $0.97(0.58-1.61)$ & $1.06(0.61-1.83)$ & $0.95(0.52-1.76)$ & $0.93(0.48-1.82)$ \\
\hline \multicolumn{9}{|c|}{$0-3$ y before index date } \\
\hline None & $60(25.2)$ & 56 (23.5) & & & & & 1 & 1 \\
\hline USS only & $13(5.5)$ & $14(5.9)$ & & & 1 & 1 & & \\
\hline AFP only & $59(24.8)$ & $69(29.0)$ & 1 & 1 & & & & \\
\hline USS + AFP & $106(44.5)$ & 99 (41.6) & $0.86(0.29-2.55)$ & $1.23(0.35-4.33)$ & $0.75(0.44-1.27)$ & $0.84(0.48-1.48)$ & $1.09(0.61-1.95)$ & $1.12(0.60-2.08)$ \\
\hline \multicolumn{9}{|c|}{$0-2$ y before index date } \\
\hline None & 77 (32.4) & $72(30.3)$ & & & & & 1 & 1 \\
\hline USS only & $15(6.3)$ & $14(5.9)$ & & & 1 & 1 & & \\
\hline AFP only & $64(26.9)$ & $75(31.5)$ & 1 & 1 & & & & \\
\hline USS + AFP & $82(34.5)$ & $77(32.4)$ & $1.67(0.40-6.97)$ & $2.07(0.44-9.66)$ & $0.63(0.34-1.16)$ & $0.77(0.40-1.47)$ & $1.15(0.63-2.09)$ & $1.18(0.63-2.23)$ \\
\hline \multicolumn{9}{|c|}{$0-1$ y before index date } \\
\hline None & $110(46.2)$ & 94 (39.5) & & & & & 1 & 1 \\
\hline USS only & $18(7.6)$ & $22(9.2)$ & & & 1 & 1 & & \\
\hline AFP only & $63(26.5)$ & $74(31.1)$ & 1 & 1 & & & & \\
\hline USS + AFP & $47(19.7)$ & $48(20.2)$ & $0.60(0.14-2.51)$ & $0.65(0.12-3.62)$ & $0.81(0.39-1.69)$ & $0.88(0.40-1.93)$ & $1.43(0.72-2.83)$ & $1.31(0.64-2.67)$ \\
\hline
\end{tabular}

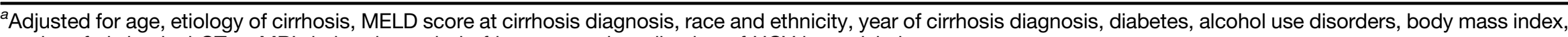
receipt of abdominal $\mathrm{CT}$ or MRI during the period of interest, and eradication of HCV by antiviral treatment. 